Gianni Belcaro is a researcher at the Cardiovascular Institute of Chieti University, Italy, a Research Fellow in Vascular Surgery at the Irvine Cardiovascular Laboratory, St Mary's Hospital, London, and Director of the Vascular and Angiology Surgical Centre, Pescara, Italy. He is the author of Laser Doppler, Vascular Screening and Venous Disorders: A Manual of Diagnosis and Treatment. He has also published several papers on the subject.

\begin{abstract}
Andrew N. Nicolaides, MS, FRCS, FRCSE, is Professor of Vascular Surgery, Honorary Consultant, and Director of the Irvine Laboratory for Cardiovascular Investigation, St Mary's Hospital Medical School, London. $\mathrm{He}$ is a member of the Vascular Society of Great Britain. His publications include Thromboembolism Aetiology: Advances in Prevention and Management (1975) and The Investigation of Vascular Disorders (1981), and a number of papers on aetiology, prevention and management of postoperative deep venous thrombosis and noninvasive cardiovascular investigation.
\end{abstract}

Gerard Stansby is Senior Lecturer and Honorary Consultant Surgeon at the Academic Surgical and Vascular Units, Imperial College School of Medicine, St Mary's Hospital, London. He is the author of over 50 academic articles. 
This page is intentionally left blank 


\title{
The Venous Clinic
}

\section{Diagnosis, Prevention, Investigations, Conservative and Medical Treatment, Sclerotherapy and Surgery}

\begin{abstract}
Gianni Belcaro
Cardiovascular Institute, Chieti University, Italy, Angiology \& Vascular Surgery, Pierangeli Clinic, Pescara, Italy,

Irvine Laboratory for Cardiovascular Investigation and Research, Department of Vascular Surgery, Division of Surgery, Anaesthetics and Intensive Care, Imperial College and St Mary's Hospital, London, U.K.
\end{abstract}

\section{Andrew N. Nicolaides}

Irvine Laboratory for Cardiovascular Investigation and Research, Department of Vascular Surgery, Division of Surgery, Anaesthetics and Intensive Care, Imperial College and St Mary's Hospital, London, U.K.

\section{Gerard Stansby}

Division of Surgery, Anaesthetics and Intensive Care, Imperial College, London, U.K.

\section{In collaboration with:}

G.B. Agus, A. Barsotti, M.R. Cesarone, D. Christopoulos, M.T. De Sanctis, S. Dhanjil, B. Eklof, C. Fisher, G. Geroulakos, G. Gizzi, G. Goren, M. Griffin, D.T.A. Hardman, M. Herny, R. Hull, L. Incandela, N. Labropoulos, A. Ledda, G. Laurora, M. Leon, A. Lennox, M. Malouf, G. Pineo, G. Ramaswami, A. Ricci, O. Thulesius, J. Vale, S. Vasdekis, M. Veller, R. Venniker, A. Zukoski and L.P. Willows 
Published by

Imperial College Press

203 Electrical Engineering Building

Imperial College

London SW7 2BT

\section{Distributed by}

World Scientific Publishing Co. Pte. Ltd.

P O Box 128, Farrer Road, Singapore 912805

USA office: Suite 1B, 1060 Main Street, River Edge, NJ 07661

UK office: 57 Shelton Street, Covent Garden, London WC2H 9HE

\section{British Library Cataloguing-in-Publication Data}

A catalogue record for this book is available from the British Library.

\section{THE VENOUS CLINIC \\ Diagnosis, Prevention, Investigations, Conservative and Medical Treatment, Sclerotherapy and Surgery}

Copyright (C) 1998 by Imperial College Press

All rights reserved. This book, or parts thereof, may not be reproduced in any form or by any means, electronic or mechanical, including photocopying, recording or any information storage and retrieval system now known or to be invented, without written permission from the Publisher.

For photocopying of material in this volume, please pay a copying fee through the Copyright Clearance Center, Inc., 222 Rosewood Drive, Danvers, MA 01923, USA. In this case permission to photocopy is not required from the publisher.

ISBN $1-86094-051-X$

Printed in Singapore. 
Dedicated to Philip Sager and Alfred Bollinger. 
This page is intentionally left blank 


\section{CONTENTS}

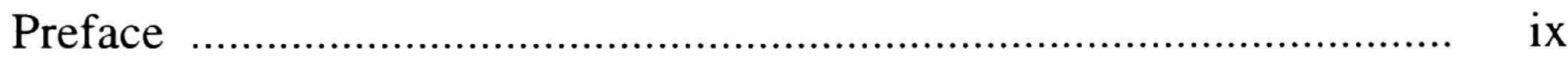

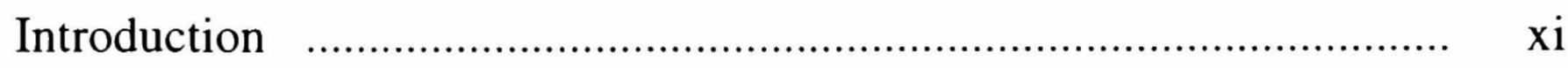

Chapter 1: The Venous System ............................................................ 1

Chapter 2: Diagnostic Tests in Venous Insufficiency ......................... 12

Chapter 3: Superficial Venous Insufficiency and Varicose Veins ............................................................. 29

Chapter 4: Superficial Thrombophlebitis .......................................... $\quad 52$

Chapter 5: Subclavian and Axillary Venous Thrombosis................... 58

Chapter 6: Chronic Venous Insufficiency and the Postphlebitic Syndrome ............................................... 66

Chapter 7: Chronic Limb Swelling …………………..................... 84

Chapter 8: Lymphatics and Lymphoedema .......................................... 88

Chapter 9: Deep Venous Thrombosis ……………….................... 100

Chapter 10: Pulmonary Thromboembolism .................................... 118

Chapter 11: Prevention of Venous Thromboembolism ....................... 126

Chapter 12: Varicocoele .............................................................. 139

Chapter 13: The Pelvic Congestion Syndrome and Vulvar Varices ......................................................... 142

Chapter 14: Haemorrhoids ............................................................ 147 
Chapter 15: Abnormal Arteriovenous Communications (Fistulas)

Chapter 16: Drugs and Treatments Used in

Venous Diseases ................................................... 156

Chapter 17: Other Venous Diseases ............................................ 168

Chapter 18: The CEAP Classification .......................................... 175

Appendix: $\quad$ Forms Used in Venous Diseases .............................. 179

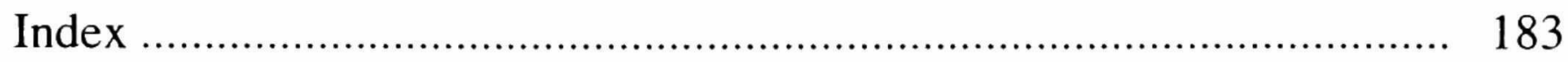




\section{PREFACE}

That veins can cause disease has been known since classical times. However, our understanding of exactly how abnormalities of the venous system cause pathology has increased enormously in the last two decades. In line with this has come an expansion in the therapeutic modalities available along with inevitable controversies as to how they should be used. The Venous Clinic aims to cover in a relatively small volume all the wide-ranging conditions which have an abnormality of the venous system as their cause, and to outline strategies and principles of investigation and treatment. We hope it will appeal to a wide range of readers in different disciplines, including surgeons and physicians, nurses, medical technologists and those involved in venous research.

Venous disease, considered collectively, imposes an enormous burden on the health resources of most developed countries. Venous ulceration alone will be experienced by approximately $1 \%$ of all adults, and pulmonary embolism remains the major cause of death after elective surgery in young patients. Such facts would seem to justify the allocation of resources to the study of venous disease. Unfortunately, in most countries venous disorders have not traditionally received the focused attention that they deserve. Perhaps this is because these diseases often cross what would be considered the usual boundaries in medical specialisation and research. Now, however, phlebology is emerging as a valid and appropriate subspeciality, with its own research journals, meetings and areas of practice. This development is more advanced in some countries than in others, but considered overall it is a rapidly growing movement. It seems beyond debate that, armed with a special interest, knowledge and understanding of the mechanisms of venous disease, phlebologists will be able to provide the best and most appropriate standard 
of care in many instances or offer advice on investigation and treatment to their colleagues in other specialities. Perhaps more important, this may lead to more and better research into venous disease and its treatment. We believe that this book will support that philosophy and hopefully, in some small way, lead to a greater appreciation of the problems suffered by patients with venous disorders.

G. Stansby

G. Belcaro

A. N. Nicolaides 


\section{INTRODUCTION}

The spectrum of venous diseases is large and much of the population is affected. Is it possible to screen patients with venous diseases and control the problem before it becomes symptomatic and expensive?

A positive answer comes from the experience of prevention of deep venous thrombosis (DVT) and pulmonary embolism (PE), which may be controlled and greatly reduced by preventive measures. However, it would also be important to know if it is possible to slow down the progression of venous insufficiency.

In this introduction we mainly consider the following problems:

- The epidemiology and screening of venous diseases;

- The evaluation of progression and of the efficacy of therapeutic measures by quality of life assessment;

- The correlation between some dietary factors (in particular, "accessory" food elements such as olive oil, fibres, berries and roots) and the incidence of venous diseases.

It is possible that these neglected aspects of venous disorders may be crucial in planning the best, most effective preventive measures and treatments to control a constellation of diseases affecting a large number of people with an otherwise normal life.

\section{Epidemiology and Costs of Venous Diseases}

Venous diseases (particularly chronic venous insufficiency and venous thromboembolism) are among the commonest problems in medicine. The precise distribution of venous diseases is largely unknown, as no prospective 
studies analysing a whole population, using noninvasive diagnostic technology (to define incompetence and/or obstruction), are available. In all developed countries varicose veins are a common problem and in European surgical wards some $30 \%$ of the nonmedical staff's time may be spent on chronic treatment of venous ulceration. Some $1 \%$ of men and $4-5 \%$ of women in Europe may have clinical venous problems. However, most statistical data result only from referrals to medical services. Therefore these figures are only partially related to the real incidence of venous diseases in the population as a whole.

Ideally only a population study including the whole population (or a defined quota sampling) may reveal the true prevalence of venous diseases including clinical and subclinical disease (for which there is no referral to medical services). A full knowledge of the prevalence of subclinical disease may give us an estimate of costs when the disease becomes symptomatic. A disease such as chronic venous insufficiency is ideal for screening, for several reasons:

(1) The prevalence in the population is high, possibly some $10 \%$ (there is no point in screening, or it is not cost-effective to screen, a population for a rare disease);

(2) The long time needed for subclinical problems (10-30 years) to progress to the clinical phase in most subjects. This long subclinical period of time is theoretically useful for avoiding the passage to clinical disease by secondary preventive measures;

(3) We have reliable and relatively low cost methods for evaluating venous problems (noninvasive investigations in venous disorders are very costeffective in revealing the extent, degree and level of venous reflux or obstruction or both).

Finally, the distribution, awareness and level of tolerance for subclinical and clinical venous diseases - such as venous insufficiency, which is not life-threatening — are extremely variable among populations, from a zero level where nutritional and basic health problems are relevant (i.e. Third World countries) to extreme, almost paranoid, attention to even minor cosmetic problems in populations (or in population strata) with high standards of living and nothing better (or worse) to fear or to think about.

It is therefore important to evaluate the distribution of venous diseases in screening a population sample in full (or at least analysing a proportional sample representing the population distribution). 


\section{The San Valentino Vascular Screening Project}

In July 1994 the San Valentino project was launched. An institute including a vascular laboratory was placed in the village of San Valentino (Central Italy, altitude $500 \mathrm{~m}$, population about 2000). The main aim of the study was to evaluate the prevalence of early, subclinical atherosclerosis in the whole population. A second project was initiated with the aim of evaluating the prevalence of venous diseases in a homogeneous population (one single valley; low level of emigration and immigration in the previous 30 years). The third part of the project was the evaluation of the progression of both arterial and venous diseases for a period of 10 years. The clinical history was recorded and subjects were clinically examined (in the standing and the supine position). Leg circumferences were measured with a tape (calf and thigh). An ATL duplex scanner was used for the ultrasound evaluation. The noninvasive investigation methods used in the project are described in detail in Chapter 2. The clinical or subclinical patterns associated with venous diseases due to incompetence were classified according to the scheme presented in Table 1. The population examined at the end of 1996 included 746 subjects without arterial disease (379 females; mean age 46.3 \pm ; range 8-94). The per cent distribution of venous diseases in this population is shown in Table 1. There was a trend for all venous diseases to increase with increasing age. The trend age/disease distribution was significantly higher for lipodermatosclerosis and ulceration. In total, $8.6 \%$ of the populations had at the time of the examination (or in their medical history) a clinically relevant venous problem. In some $3 \%$ within this group these problems were severe (they had been treated or were under treatment or had required hospital admission). In a further $2.8 \%$ the venous problem was less severe (not requiring treatment) or subclinical.

The use of medical products and treatments in these patients is indicated in Table 2. The average cost per patient for a year's treatment was $850 \mathrm{ECU}$, including hospital costs, lost days of work and medical products. Some $45 \%$ of these costs had been charged to the community services and hospitals (the health community) and the rest had been directly charged to the patients. We were able to (arbitrarily) calculate that more than $50 \%$ of the services, treatments and products used had been either inappropriate or ineffective. Considering that venous diseases are usually chronic, the community costs make these diseases a major health care problem. Inefficiency, lack of 
Table 1. Clinical and subclinical venous patterns. The clinical or subclinical patterns associated with venous diseases of the lower limbs were classified according to the following scheme. The per cent of the population with each venous problem is shown. In $5.5 \%$ of the subjects more than one problem was present (i.e. teleangectasias and varicose veins).

\begin{tabular}{ll}
\hline \multicolumn{1}{c}{ Category } & $\%$ \\
\hline 1. Teleangectasias & 8 \\
2. Varicose veins (no signs/symptoms or complications) & 6 \\
3. Varicose veins with mild initial complications (skin changes) & 2 \\
4. Severe superficial and/or deep incompetence & \\
(including chronic veins and severe complications, & 3.3 \\
i.e. thrombophlebitis, bleeding, skin changes) & 2 \\
5. Chronic venous hypertension & 2 \\
6. Small (<lcm ${ }^{2}$ ), initial (first) ulcerations & 1 \\
7. Severe, recurrent ulcerations with infection & 4 \\
8. Previous DVT (history only) $\quad$ (documented) & 1 \\
9. Previous PE (history only) & $<1$ \\
10. Thrombophlebitis (history only) & 0.2 \\
\hline \hline
\end{tabular}

Table 2. Products used for the treatment of venous problems and per cent of patients with venous disease using them acutely ( $\mathrm{AC}$ : once-twice) or chronically $(\mathrm{CH}$ : continuously for more than three months). More than one treatment was or has been used by some $9 \%$ of patients.

\section{Products used for treatment}

\begin{tabular}{lcc}
\hline & $\mathrm{AC}$ & $\mathrm{CH}$ \\
\cline { 2 - 3 } 1. OTC products (or any treatment not requiring prescription) & 18 & 7 \\
2. Specialized drug (for venous diseases) & 21 & 12 \\
3. Compression & 24 & 11 \\
4. Surgery (any type of surgical treatment) & 12 & - \\
5. Sclerotherapy & 9 & 3 \\
6. Combined treatments (i.e. sclerotherapy and surgery) & 9 & 8 \\
7. Alternative treatments (herbal products etc.) & 10 & 3 \\
\hline \hline
\end{tabular}


prevention and differences of standards possibly double the costs of venous diseases.

In conclusion, many of the population are affected by clinical venous problems and subclinical problems, which results in major costs for the health services of developed countries.

Whether screening of the youngest part of the population for venous problems before they become clinically relevant (and expensive) can be effective in reducing costs and controlling the evolution of many venous diseases is still to be evaluated. It is possible that in many subjects with early venous disease the control of progression and evolution to clinical stages may be obtained with simple preventive methods. Knowing correctly the sites and degree of incompetence, it is possible to suggest an evolution controlling plan using the several methods available (sclerotherapy, selective surgery, compression or medical treatment).

\section{Quality of Life and Venous Diseases}

Attempts to quantitatively evaluate venous diseases, their progression and the effects of treatment are a major problem in the venous clinic. The evaluation of ambulatory venous pressure (AVP) or air plethysmography (APG) parameters is useful for quantifying and evaluating the effects of treatment in groups of subjects but is complicated and time-consuming and does not always reflect the point of view of the single patient. From a patient's point of view the quality of life $(Q O L)$, or its deterioration associated with the venous problem, is the most important criterion to consider. What is the point of changing AVP when the patient does not change his standard of life (i.e. requiring less medical consultation time, less cost for taking care of the problem and a more active life)? For a patient it is more important to be able to shop or to walk and buy a paper without help or difficulty than to undergo a purely physiological change (i.e. in venous pressure).

For a list of ten medical outcome QOL items (Table 3) an arbitrary qualityof-life score concerning venous problems may be considered. The total score varies between 0 and 100 (100 indicates a 100\% fit subject). The decrease in the score indicates a deterioration in the patient's life due to any venous disease. If we consider a group of symptomatic subjects (89) with superficial 
Table 3. Medical outcome in venous diseases: quality of life score.

\begin{tabular}{l}
\hline \hline 1. Mobility \\
2. Pain \\
3. Swelling \\
4. Subjective symptoms \\
5. Objective signs \\
6. Independence \\
7. Working life \\
8. Social life \\
9. Cosmetic aspect \\
10. Costs (in time and money) \\
Total $0-100$ \\
$100=100 \%$ fit \\
\hline \hline
\end{tabular}

CORRELATION QOL SCORE/AVP

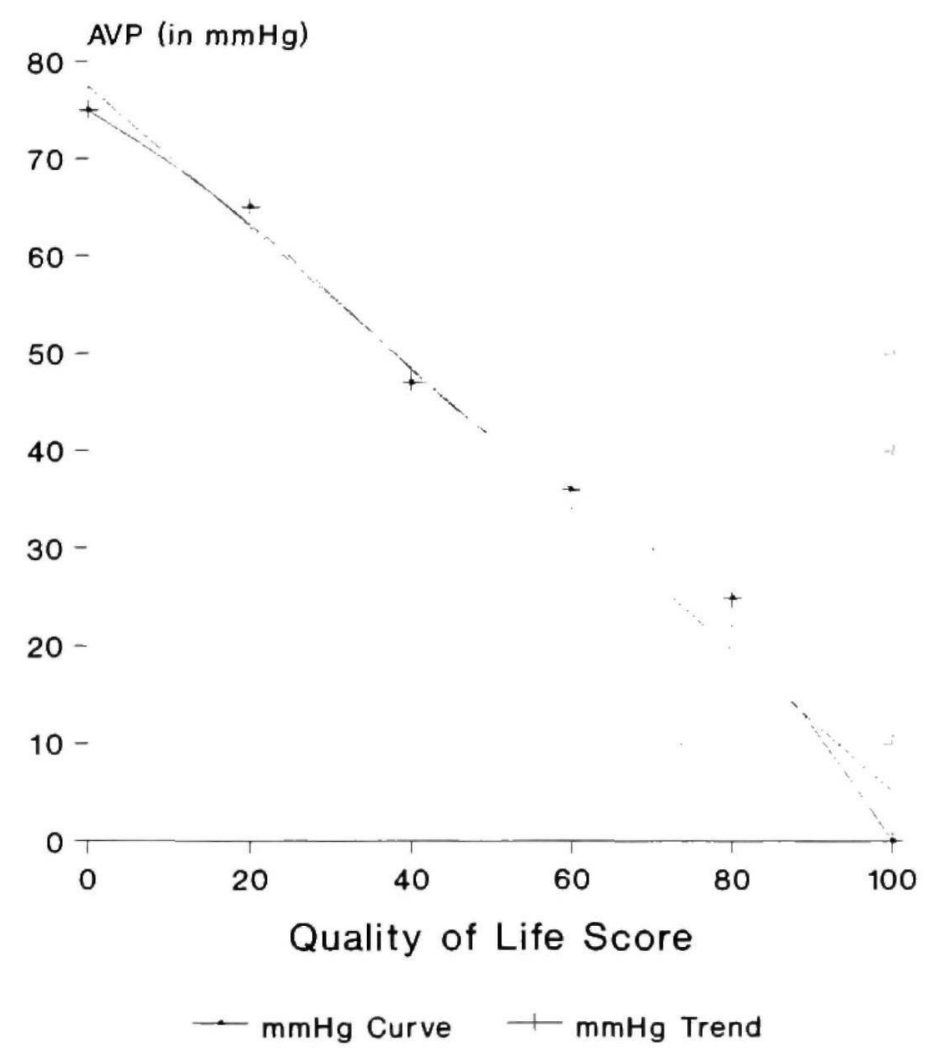

$100 \cdot$ normality

Figure 1. Inverse correlation between the QOL score and the AVP measurement. This finding indicates that physiological and QOL assessments are in some way comparable. 
venous incompetence (35\%), deep venous incompetence without (30\%) and with $(5 \%)$ ulcerations and with mixed deep+superficial incompetence, it is possible to observe an inverse correlation between the QOL score and the AVP measurement (Figure 1). This confirms that physiological and QOL assessments are in some way comparable.

The correlation between years of venous diseases and QOL score shown in Figure 2 indicates a progressive deterioration of the QOL with the persistence of the venous disease and suggests that treatments useful for stopping or slowing down the progression of venous diseases may also act on the progressive deterioration of the QOL.

In a different series of 104 patients with symptomatic superficial and/or deep venous disease, it is possible to observe (Figure 3 ) that a decrease in AVP (after treatment) is inversely related to an increase in the QOL score (after treatment). These measurements of AVP and QOL were repeated 12 months after the appropriate treatment (surgery and/or sclerotherapy).

It is clear that there is a correlation, not always precise, between physiological parameters of venous insufficiency (i.e. AVP) and quality of

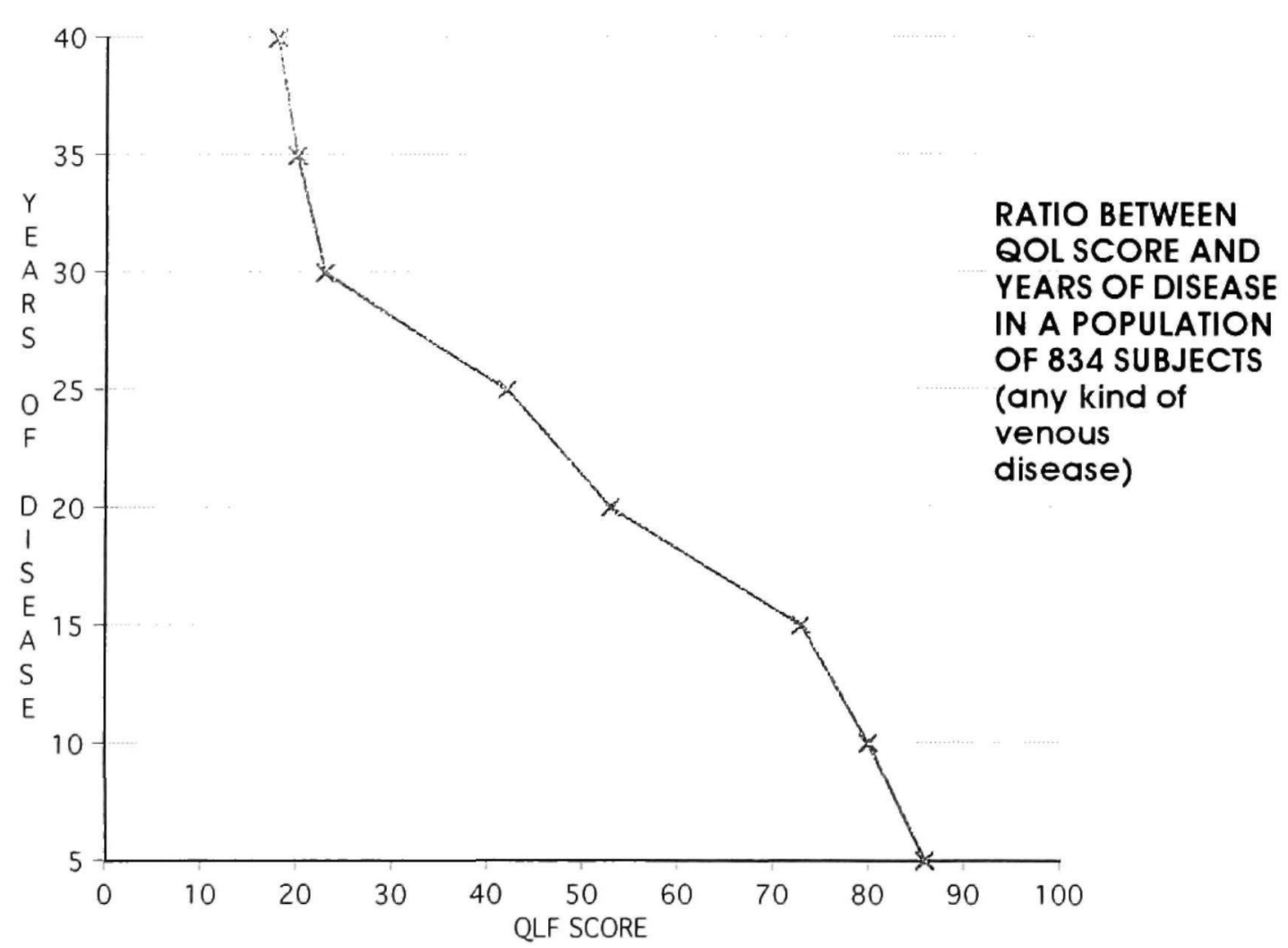

Figure 2. The correlation between years of venous diseases and QOL score shown. The graph indicates a progressive deterioration of the QOL with the persistence of the venous disease. 


\section{QOL SCORE AND PRESSURE VARIATIONS}

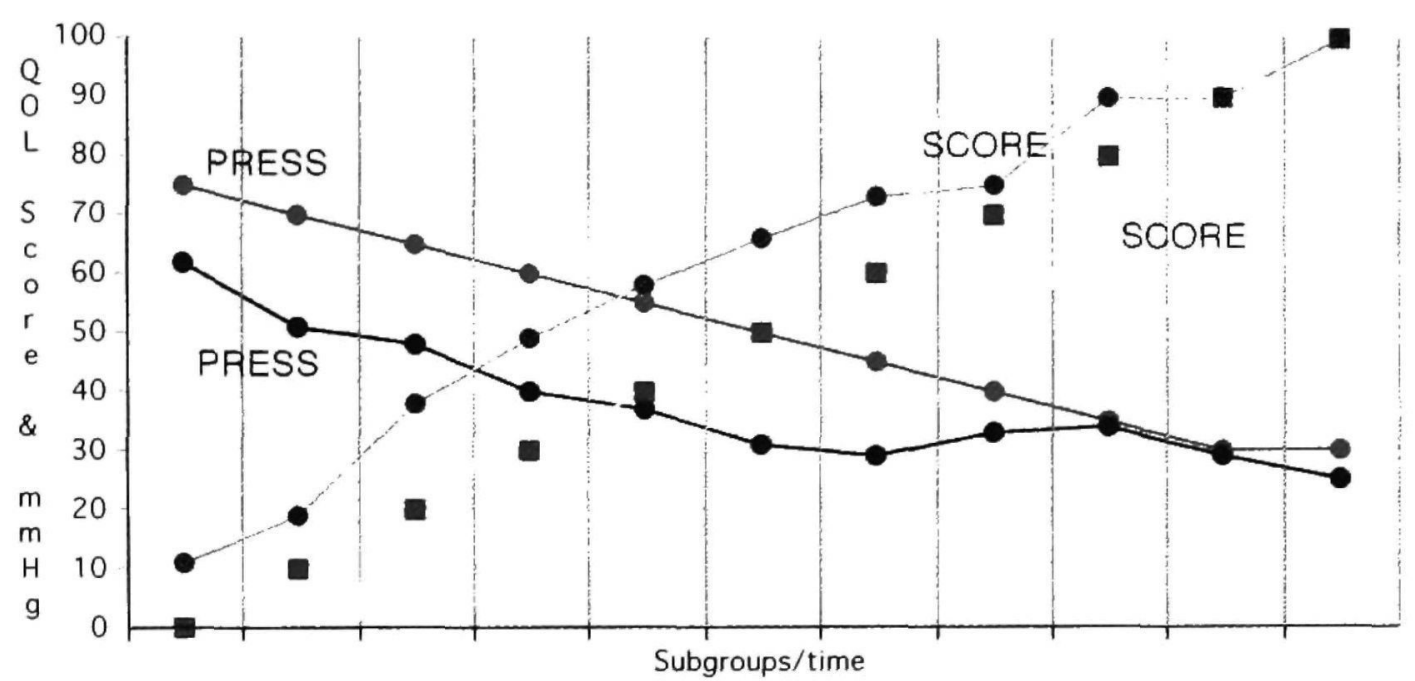

AVP before

aVP after

$\square$ QOL score after

QOL score before

Figure 3. In a series of 104 patients with symptomatic superficial and/or deep venous disease it was possible to observe that a decrease in AVP (after treatment) was inversely related to an increase in the QOL score. The measurements of AVP and QOL were repeated 12 months after the appropriate treatment (surgery and/or sclerotherapy).

life. The future evaluation of treatments and management procedures of venous disorders must consider this new evaluation approach.

Finally, there is possibly an important correlation between diet and vascular diseases. Whilst in the past attention has been focused on the arterial side, there is now some evidence suggesting that diet may also be involved in the genesis of some venous disorders. If we observe (in a sample of 400 subjects) the consumption of some "accessory food elements" i.e. olive oil, berries and roots, and fibre content, we can observe a very interesting trend. There is an increase in the incidence of venous diseases (including haemorrhoids) and the consumption of these three elements. Whether they may have direct effects, particularly in the growth phase, or they are just a marker or expression of a different quality of life, it is difficult to say at the moment.

In particular, roots and berries, a very common "accessory" food in many rural areas - where they are directly collected from the ground - contain many vitamins, flavonoids (in the pigmented part of the plants) and other pharmacologically interesting compounds (including some alkaloids). They (Figure 4) appear to be connected with the evolution of venous diseases and their consumption appears to be inversely correlated with the occurrence of venous diseases. 


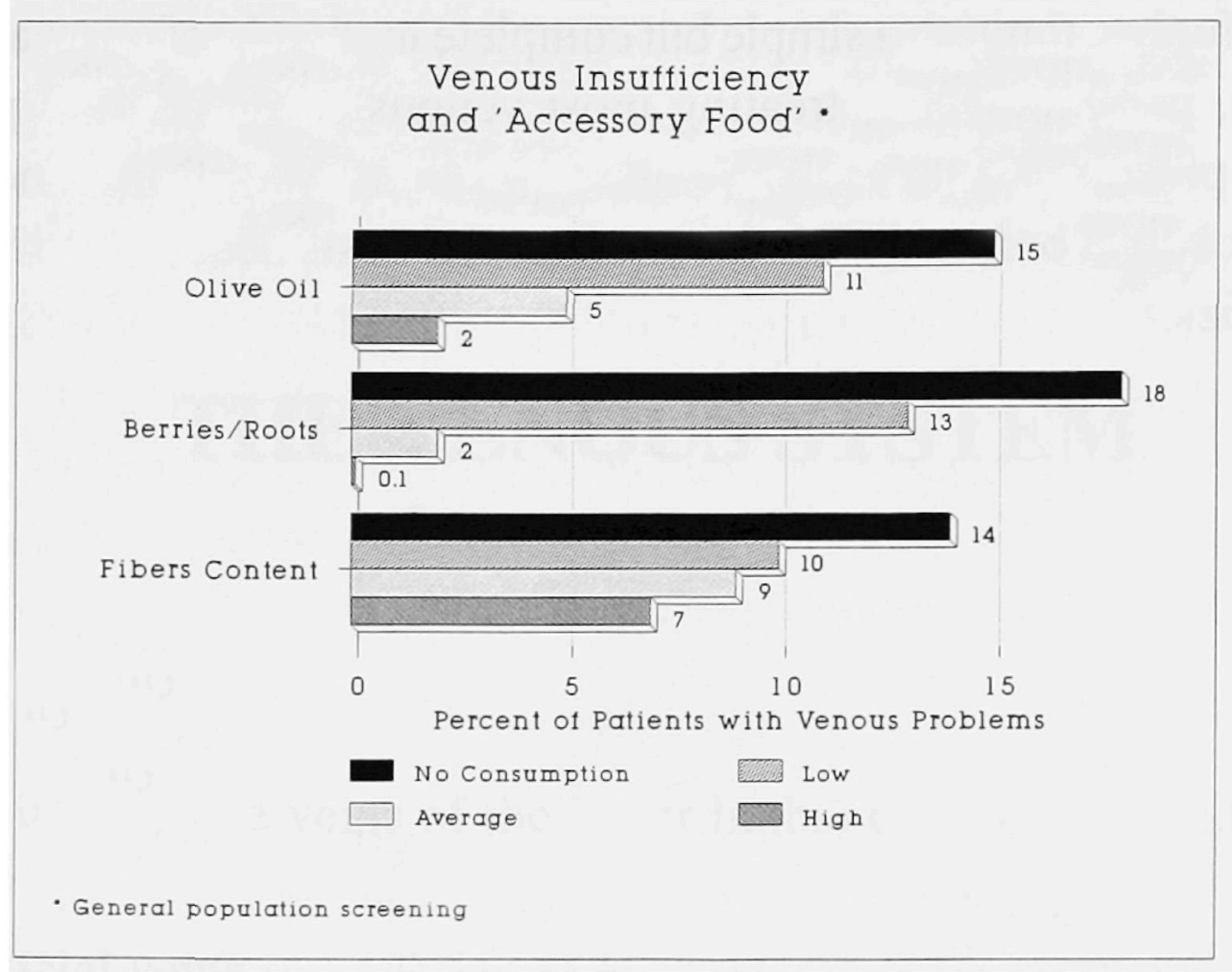

Figure 4. Olive oil, fibres and particularly roots and berries - a common "accessory" food in many rural areas - contain many vitamins, flavonoids (in the pigmented part of the plants) and other pharmacologically interesting compounds (including some alkaloids). They appear to be connected with the evolution of venous diseases and their consumption appears to be inversely correlated with the occurrence of venous diseases (including haemorroids).

Burkitt's observation that the content of fibres in the diet may be inversely proportional to the occurrence of some venous disorders may have followed this line of speculation. However, the inverse correlation of fibre intake and venous disease is less clear in our study.

In conclusion, venous disorders are common and tend to alter the quality of life of many otherwise healty subjects for a long period of time. New, noninvasive investigation methods useful for screening, the evaluation of the quality of life in subjects with venous diseases, and some new, interesting considerations (i.e. diet elements) are interesting options for the research in this field in the next few years. The preservation of a healthy venous system and preventing early and progressive deterioration is an important aim. It is now possible to avoid reaching the symptomatic stage by controlling most venous diseases in the subclinical phase. Whether this is possible at acceptable social costs will be the aim of our future clinical research. 
The diffusion, through a simple but complete textbook, of our way of seeing, evaluating, preventing and treating most venous disorders will possibly be useful for promoting more attention to these common - often neglected clinical problems before their human and social costs become too high for our communities. 